\title{
Umbrella Wheel: A Stair-Climbing and Obstacle-Handling Wheel Design Concept
}

\author{
Simon Iversen, Jerome Jouffroy
}

\begin{abstract}
This paper presents a design for stair-climbing using a wheel that can split into segments and walk up stairs or surmount other obstacles often found where humans traverse, while still being able to retain a perfectly round shape for traveling on smooth ground. Using this change of configuration, staircases with a wide range of dimensions can be covered efficiently and safely. The design, named Umbrella Wheel, can consist of as many wheel segments as desired and as few as two. A smaller or higher number of wheel segments has advantages and disadvantages depending on the specific situation. Modeling the trajectory of the wheel when as it ascends or descends stairs is given and the results are analyzed.
\end{abstract}

Keywords-Locomotion, mechanics, mechatronics, mobile robotics, obstacle handling, path modeling, stair-climbing.

\section{INTRODUCTION}

V EHICLES such as autonomous robots or guided vehicles can have a large variety of mechanisms to provide movement, such as wheels or caterpillar tracks. The efficiency of travel, the smoothness of locomotion and the ability to handle obstacles are among the criteria that can determine which solution fits best for a specific situation. For some applications, production considerations can put a constraint on the complexity level, as a low number of degrees of freedom generally makes control easier. For vehicles that should operate in locations designed for people, stair-climbing can be a challenge for vehicles, since stairs are designed for the bipedal movement of a human and not for the mechanically simpler motion of wheels.

The stair-climbing problem has been under consideration for many years. For hand trucks, earlier designs can, at least, be traced back to as far as 1882 [1], where a set of wheels placed around a rotating frame allows the vehicle to ascend or descend a stair-case. Variations on this design are still among the most popular solutions today (see for example [2]). An alternative but common solution in the field of robotics is to use caterpillar tracks [3], which has the advantage of good handling on most types of difficult terrain, though at the cost of low efficiency on even ground when compared to a wheel. Furthermore, this design can also lead to damage of the stairs on the edge of the step. To prevent damage to the environment, a design for a stair-climbing wheelchair has been developed [4], with however considerable additional weight. Other solutions obviously leg-based designs, and these are known to be able to handle any terrain where a stable footing can be achieved, mostly these have four [5] or more legs [6]. However, these are generally and comparatively quite

Simon Iversen and Jerome Jouffroy are with the Mechatronics Section and the Mads Clausen Institute at the University of Southern Denmark (e-mail s_iversen@mci.sdu.dk and jerome@mci.sdu.dk). complicated to construct and control. Flying drones have no problem handling stairs, but lack efficiency, are noisy and cannot handle heavy cargo. Finally, some vehicles separate the stair-climbing from general locomotion to achieve the ease and efficiency of wheeled travel while still being able to overcome stairs, for example by having rotating arms that can lift the vehicle onto a step [7], this allows for simpler mechanisms since it no longer has to handle general locomotion.

In search of a good stair-climbing solution, a new design for a wheel that allows for smooth and efficient locomotion on even ground as well as the possibility to ascend and descend stairs is presented in this paper. This design, referred to as Umbrella Wheel, consists in having the wheel spread out using arms that can grab onto obstacles, such as stairs, and overcome them.

After this introduction, in Section II describes the concept along with variations as well as a small discussion on the obstacles that the Umbrella Wheel can overcome. The following section investigates the stair-climbing motion and looks into path modeling. Section IV presents a short case study with a few examples related to path evolution, showing differences between variations of the wheel design. Section V describes certain aspects of the design that can affect implementation, while brief concluding remarks end the paper.

\section{CONCEPT}

The simple idea behind the Umbrella Wheel concept consists in keeping a perfectly round wheel for travel on smooth ground and then transform the wheel in order to give it the capability of overcoming obstacles such as stairs when necessary. Our design works by having the outer part of the wheel being able to separate into several pieces, referred in the following as wheel segment, each of which extending outwards from the axle to form spokes.

A 3D model of the Umbrella Wheel design in a half-way position is shown in Fig. 1. Fig. 2 shows sideway views of the wheel in the fully closed and fully open configuration. Note that these $3 \mathrm{~d}$ models have been kept at the same scale in order to give an idea of the magnitude of the expansion of the wheel segments.

In order to see more precisely how the concept works, let us go back to Fig. 1, where the main axle of the wheel (labelled " 1 " in the figure) is connected with two parts (in green). The first one is fixed (labeled "2") is fixed to the axle, while the other (i.e. piece " 3 ") is free to slide along it, and in doing so extends or retracts the arms ("5") and with them the wheel segment rims ("4"). As one of the possible ways to extend 


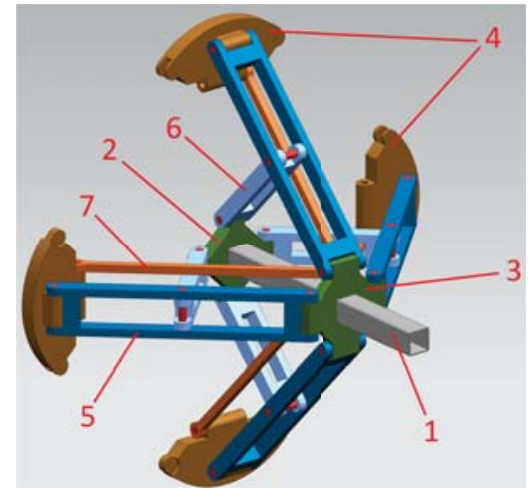

Fig. 1 Isometric view of the Umbrella Wheel concept in midway position

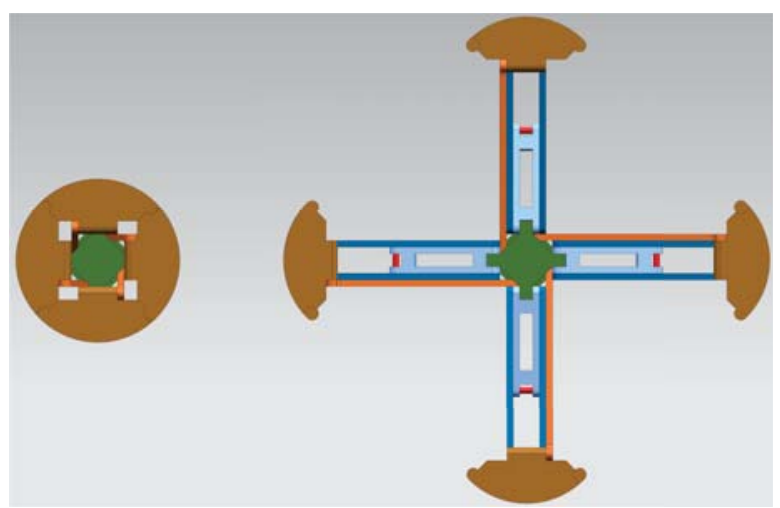

Fig. 2 Umbrella Wheel in the closed(left) and open(right) positions

or retract the arms, the current configuration uses the parts labeled " 6 " to lift up the arms, this in a fashion similar to an umbrella, hence the name of the proposed concept. Note that by having the short arms be half the length of the long arms, there is no movement of the wheel segments in the direction of the axle when the arms extend. Hence the wheel segment rims move only radially away from or closer to the axle. The orange parts (labeled "7") ensure that the wheel segments keep their orientation fixed by creating a parallelogram. Thus, the strength of the design lies in its relative simplicity. Indeeed, the location of the driving force, ie the axle, is essentially the same, no matter what the current geometry of the wheel is. The additional variable is simply the geometry of the wheel. Additionally, the rim of each wheel segment is quite generic and many different types of materials and thread profile can be used (for example rubber) to ensure proper traction.

\section{A. Number of Wheel Segments}

The Umbrella Wheel concept works with as few as two wheel segments and up to as many wheel segments as the designer would like. There are obviously both advantages and disadvantages to having more or fewer segments, making the ideal number different for each situation. A higher number of segments gives generally smoother stair travel, while a lower number of segments needs fewer parts and requires less arm extension to climb the same stair. Conversely, a larger number of segments allows for a larger wheel in the closed position while fewer segments can require a smaller closed-wheel size. Implications of the design choices are investigated further in Sections IV and V. Pictures of the 3D model for a three-segment Umbrella Wheel can be seen in Fig. 3.

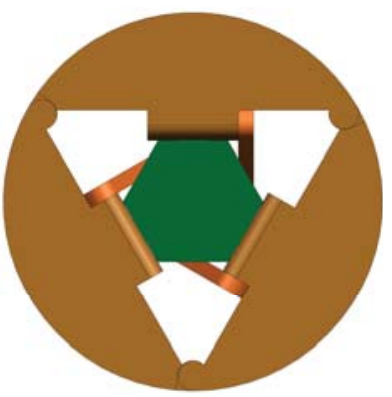

(a) Closed position

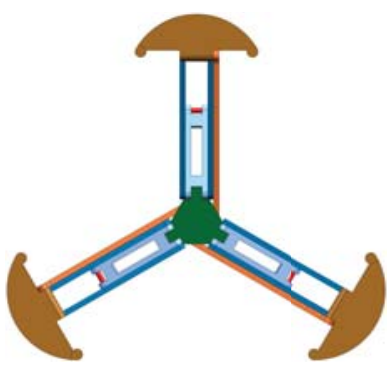

(b) Open position

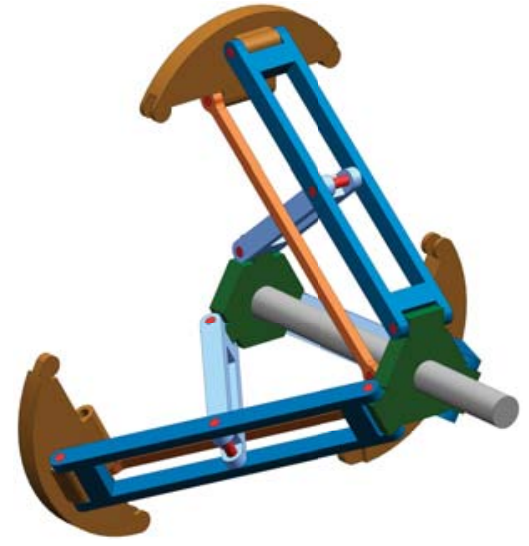

(c) Isometric view

Fig. 3 Umbrella Wheel with three leg segments

\section{B. Overlapping of Wheel Segment Rims}

Having the wheel segment rims divided into strict partitions result in the rims ending in sharp corners, which will serve as the point of contact on the stairs. A sharp corner can result in high contact pressure that will potentially damage the steps of the stairs, while the corner itself could wear over time. In turn, this wear could also lead to an altered and imperfect circle when the wheel in closed configuration. To improve upon this, an overlapping geometry was introduced at the corners of the wheel segment rimes. The overlap continues the outer curve tangentially to the curve of each rim (the overlap is made circular for design simplicity). For comparison, the two kinds of corners (rims sharp corners and corners with overlap) are represented in Fig. 4.

\section{Different Obstacles}

While the Umbrella Wheel concept was introduced with stair-climbing in mind, it can also handle other kinds of obstacle such as open gaps, and even ladders.

In the case of open gaps, the gap distance the wheel can cover depends on the possible extension of the wheel segments. For the largest reach, two opposite wheel segments 


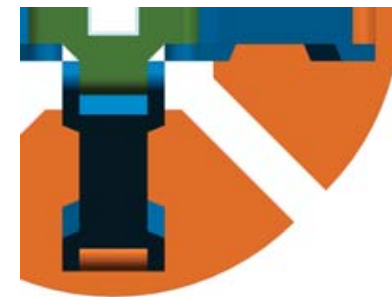

(a) Without overlap

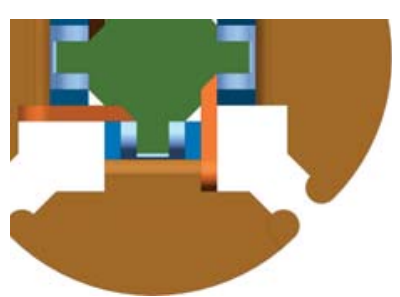

(b) With overlap
Fig. 4 Close-up of rim corner overlap

will be in contact with the ground (on either side of the gap) as seen in Fig. 5.

For climbing ladders the process is slightly more involved as it requires changing the extension during the climb. To climb a ladder, when already positioned on a rung, the next wheel segment is positioned above the rung of the ladder and then the extension of the segments is lowered, thereby placing the piece on the rung and raising the previous segment into the air. The wheel is rotated and once the previous piece is clear of the previous rung, the extension is raised again to allow the next segment to get into position above the next rung of the ladder. This process is repeated for each rung of the ladder. Additionally, it is important for ladder-climbing that the body of the vehicle is capable of ensuring the orientation of the wheel with respect to the ladder; which is ensured if the body extends at least as far back as two rungs of the ladder plus the required extension of the arm when climbing the ladder.

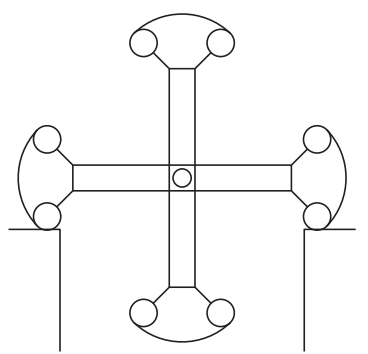

Fig. 5 Umbrella Wheel crossing a gap

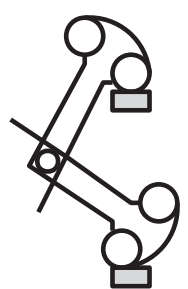

Fig. 6 Umbrella Wheel climbing a vertical ladder

\section{PATH Modeling}

In order to have more insight into the motion induced by the Umbrella Wheel, we have developed a mathematical model to calculate its path on a standard staircase. The knowledge

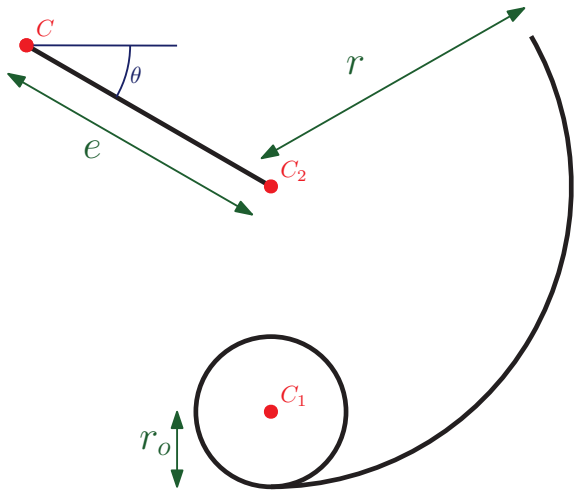

Fig. 7 Points for modeling

gained from modeling can help inform on different design choices and their associated performance. Modeling the path of the wheel also allows for comparison with other stair-climbing designs, such as the satellite wheel mechanism analyzed in [8].

To model the motion of the Umbrella Wheel, three points have been defined as shown in Fig. 7. The center-point of the wheel, noted $C$ with coordinates $\left(C_{x}, C_{y}\right)$, the center of the active wheel segment, $C_{2}=\left(C_{2_{x}}, C_{2_{y}}\right)$, and the center point of the corner overlap of the active wheel piece, $C_{1}=$ $\left(C_{1_{x}}, C_{1_{y}}\right)$.

The movement of the wheel when ascending or descending stairs goes through two phases: rolling on the corner of the segment rim as in Fig. 8a and rolling on the segment rim itself as in Fig. 8b. The arrows seen in both figures show the direction of travel for the above-mentioned points. For a four-segment Umbrella Wheel, a quarter of a rotation is needed for each step, for a three-segment wheel a third of a rotation is needed, etc. after which the motion is repeated for the next step.

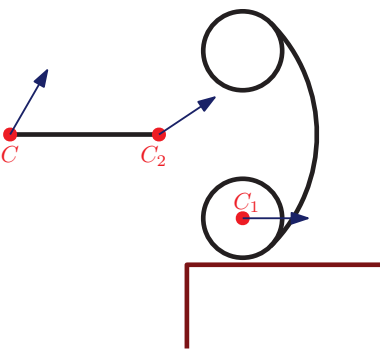

(a) Rolling on corner

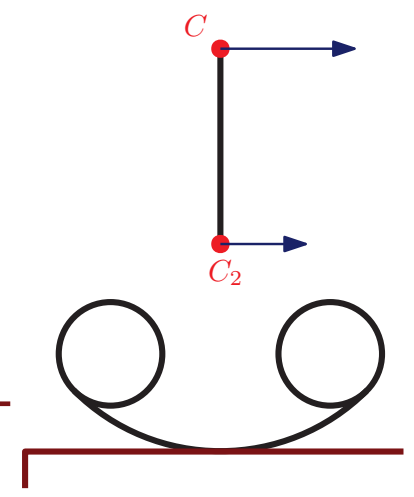

(b) Rolling on rim
Fig. 8 Movement of points for path modeling

Using the angle of the wheel segment with respect to the horizontal, noted $\theta$, also shown in Fig. 7 and the position of $C_{1}$, the position of point $C_{2}$ is described by

$$
\begin{aligned}
& C_{2_{x}}=C_{1_{x}}-\cos \left(\frac{\pi}{n}+\theta\right) \cdot\left(r-r_{0}\right) \\
& C_{2_{y}}=C_{1_{y}}+\sin \left(\frac{\pi}{n}+\theta\right) \cdot\left(r-r_{0}\right)
\end{aligned}
$$


where $r_{o}$ is the radius of the overlap part of the wheel segment rim and $n$ is the number of wheel segments.

From the angle $\theta$ and the position of $C_{2}$, the coordinates of $C$ are given by

$$
\begin{aligned}
& C_{x}=C_{2_{x}}-\cos \theta \cdot e \\
& C_{y}=C_{2_{y}}+\sin \theta \cdot e
\end{aligned}
$$

with $e$ being the arm extension of the Umbrella Wheel (see Fig. 7).

The Umbrella Wheel only makes contact with the ground or tread of the steps, and hence can be shifted horizontally without changing the motion, as long as the vertical part of the stair (ie the riser) is not interfering with the wheel. The starting point has been chosen such that $C_{1_{x_{0}}}=r_{o}$ with the edge of the step being at 0 .

If angle $\theta$ verifies $\theta \leq \frac{\pi}{2}-\frac{\pi}{n}$, the movement is in the first phase and the position of $C_{1}$ can be calculated as

$$
\begin{gathered}
C_{1_{x}}=\left(\theta-\theta_{0}\right) \cdot r_{o}+C_{1_{x_{0}}}, \quad C_{1_{x_{0}}}=r_{o} \\
C_{1_{y}}=R+r_{o}
\end{gathered}
$$

From these values, the total position of the wheel can be calculated using (1)-(4).

When $\theta$ verifies $\theta>\frac{\pi}{2}-\frac{\pi}{n}$ the movement is in the second phase. The position of $C_{2}$ in the $x$ (horizontal) direction is the angle traveled in phase two times the radius of rotation plus the $x$ position at the start of the second phase. At the start of the second phase $C_{1_{x}}=C_{2_{x}}$, which gives

$$
\begin{gathered}
C_{2_{x}}=\left[\theta-\left(\frac{\pi}{2}-\frac{\pi}{n}\right)\right] \cdot r+\left[\left(\frac{\pi}{2}-\frac{\pi}{n}\right)-\theta_{0}\right] \cdot r_{o}+C_{1_{x_{0}}} \\
C_{2_{y}}=R+r
\end{gathered}
$$

Combining (1)-(8) and using the constant

$$
\phi=\frac{\pi}{2}-\frac{\pi}{n}
$$

gives the following equations for the center point of the wheel.

$$
\begin{aligned}
& C_{x}=\left\{\begin{array}{rlrl}
\left(\theta-\theta_{0}\right) & \cdot r_{o}+C_{1_{x_{0}}} & & \\
& -\cos \left(\frac{\pi}{n}+\theta\right) \cdot\left(r-r_{o}\right) & & \theta \leq \phi \\
& -\cos \theta \cdot e, & & \\
(\theta-\phi) & r & & \\
& +\left(\phi-\theta_{0}\right) \cdot r_{o} & & \\
& +C_{1_{x_{0}}}-\cos \theta \cdot e, &
\end{array}\right. \\
& C_{y}= \begin{cases}R+r_{o}+\sin \left(\frac{\pi}{n}+\theta\right) \cdot\left(r-r_{o}\right) & \theta \leq \phi \\
R+r+\sin \theta \cdot e, & \theta>\phi\end{cases}
\end{aligned}
$$

with

$$
\theta \in\left[\theta_{0}, \frac{2 \pi}{n}+\theta_{0}\right]
$$

where $\theta_{0}$ is the starting angle, calculated in the section below, $R$ is the rise height of the stair-step, while $C_{1_{x_{0}}}$ is the starting $x$ position of the point $C_{1}$.

\section{A. Starting Angle}

The starting offset angle for the simulation, $\theta_{0}$, is obtained using the following equation. The starting angle is observed when the wheel is in contact with both steps of the stairs as can be seen in Fig. 9. Hence we have

$$
\begin{aligned}
\theta_{0}= & \frac{\pi}{2}-\frac{2 \pi}{n}+\cos ^{-1}\left[\frac{R-\left(r-r_{o}\right)}{l}\right] \\
& -\tan ^{-1}\left[\frac{e \cdot \sin \left(\frac{2 \pi}{n}\right)+\left(r-r_{o}\right) \cdot \sin \left(\frac{\pi}{n}\right)}{e-e \cdot \cos \left(\frac{2 \pi}{n}\right)-\left(r-r_{o}\right) \cdot \cos \left(\frac{\pi}{n}\right)}\right]
\end{aligned}
$$

where

$$
\begin{aligned}
l= & \left(\left[e \cdot \sin \left(\frac{2 \pi}{n}\right)+\left(r-r_{o}\right) \cdot \sin \left(\frac{\pi}{n}\right)\right]^{2}\right. \\
& \left.+\left[e-e \cdot \cos \left(\frac{2 \pi}{n}\right)-\left(r-r_{o}\right) \cdot \cos \left(\frac{\pi}{n}\right)\right]^{2}\right)^{1 / 2}
\end{aligned}
$$

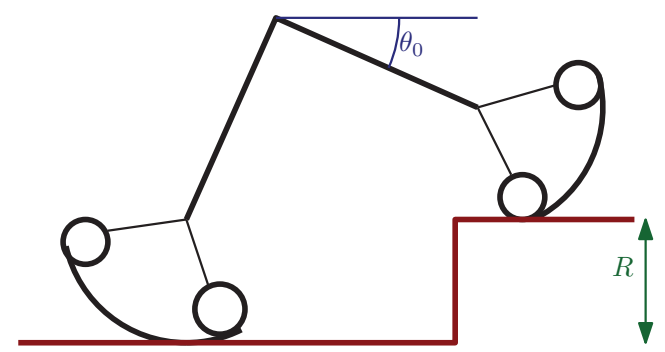

Fig. 9 Starting position for the simulation

\section{B. Calculating the Arm Extension}

Finding the proper arm extension for a given stair cannot be found directly through an analytical function. Instead a numerical answer can be found by searching for an extension that gives the correct step ground. Using the $l$ from (12) and $\phi$ from (9).

$$
\begin{aligned}
G= & \cdot \sqrt{1-\left[\frac{R-\left(r-r_{o}\right)}{l}\right]^{2}} \\
& +r_{o} \cdot\left(\phi-\theta_{0}\right)+r \cdot\left(\theta_{0}+\frac{2 \pi}{n}-\phi\right)
\end{aligned}
$$

In Fig. 10 the stair ground is set to be $G=285 \mathrm{~mm}$ and the overlap radius is in both cases set to be $r_{o}=10 \mathrm{~mm}$. The graph shows the needed extension for wheels of radii $r=100 \mathrm{~mm}$ and $r=125 \mathrm{~mm}$. The graph is the result of numerical solutions to (13). The graph shows that for a given set of stairs a smaller wheel radius will require a larger extension. Additionally, a taller step height will also require a larger extension.

\section{CASE STUdies}

\section{A. Number of Wheel Segments}

Several configurations of the Umbrella Wheel design have been simulated using the path modeling described in Section III. These simulations illustrate how the number of 


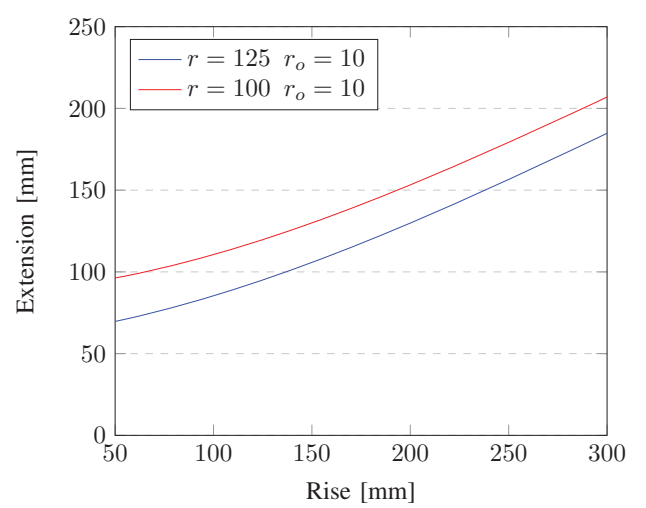

Fig. 10 Relationship between step height and required extension

wheel segments affect the path of the vehicle when ascending or descending stairs. Fig. 11a shows the path model of a two-segment Umbrella Wheel, Fig. 11b shows the path of a three-segment Umbrella Wheel, Fig. 11c four segments and Fig. 11d reprents a wheel with 6 wheel segments. For all of the associated paths, the stair dimensions have been kept constant to allow for proper comparison, with parameter values $R=160 \mathrm{~mm}, G=285 \mathrm{~mm}$ and $r=75 \mathrm{~mm}$.

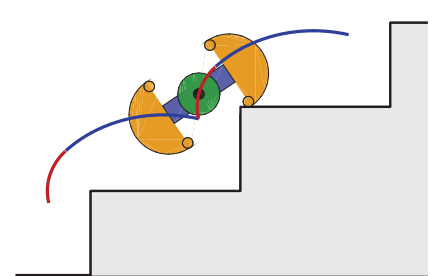

(a) Wheel with two segments

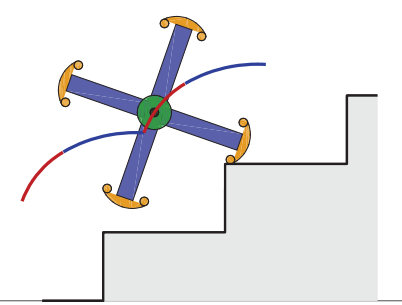

(c) Wheel with four segments

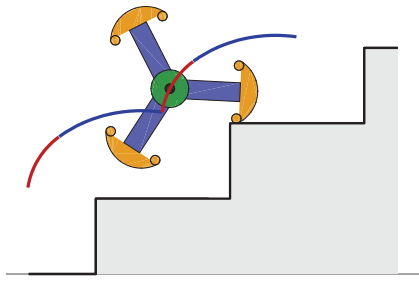

(b) Wheel with three segments

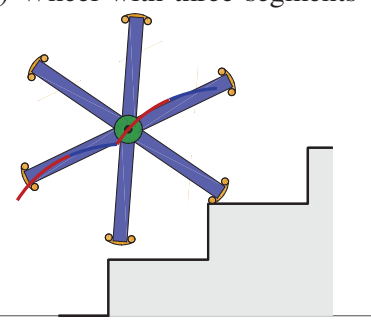

(d) Wheel with six segments
Fig. 11 Path modeling of different configurations (Phase 1 red, Phase 2 blue)

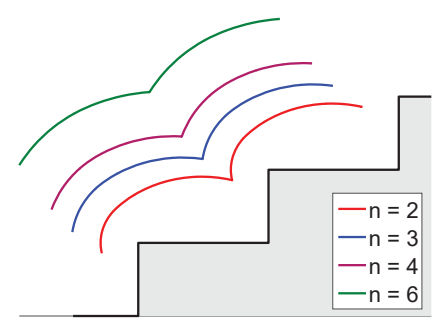

Fig. 12 Path models from different number of segments

The graphs in Fig. 11 show that the path of the vehicle is smoother for wheels with more segments as evident from the angle at the transition from one step to the next. The figures also show that a higher number of wheel segments result in the vehicle being further from the staircase due to the higher extension needed.

In Fig. 12 the paths of all four configurations are shown together.

\section{B. Wheel Radius}

The behavior of the Umbrella Wheel with different radii have also been simulated. Fig. 13a shows the path of an Umbrella Wheel with a smaller wheel segment radius, $r=$ $50 \mathrm{~mm}$, and Fig. 13b shows the path model of an Umbrella Wheel with a larger one, $r=125 \mathrm{~mm}$. The stair dimensions are the same for both simulations (with $R=160 \mathrm{~mm}$ and $G=285 \mathrm{~mm}$ ).

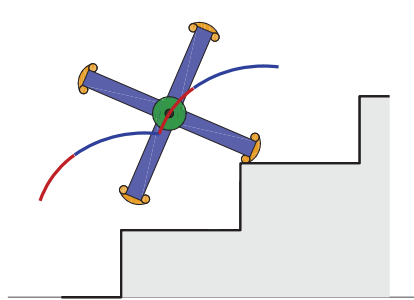

(a) Wheel with small radius

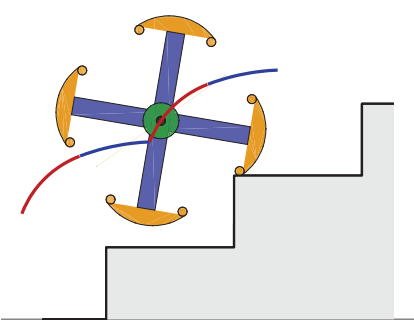

(b) Wheel with large radius
Fig. 13 Path modeling of different radii

As can be seen in Fig. 13, the radius of the wheel does not have a large impact on the smoothness of the vehicle path, though the extension needed is significantly less for the large radius wheel than for the small-radius wheel, as also seen in Fig. 10.

\section{Step Height}

The variable extension allows one wheel configuration to adapt to fit a wide range of stair dimensions, path models have been simulated for a wheel on two different stairs. Fig. $14 \mathrm{a}$ shows the path of an Umbrella Wheel climbing a staircase with a low rise, $R=85 \mathrm{~mm}$, while Fig. $14 \mathrm{~b}$ shows the path of that same Umbrella Wheel climbing a staircase with a high rise, $R=250 \mathrm{~mm}$. All other parameters are kept the same, with $r=100 \mathrm{~mm}$ and $G=285 \mathrm{~mm}$.

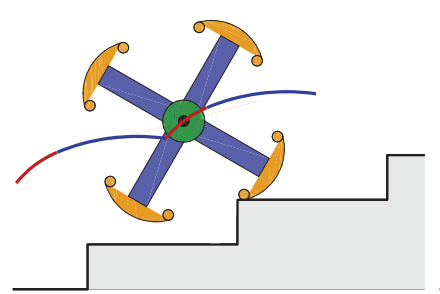

(a) Path of Umbrella Wheel on low stairs

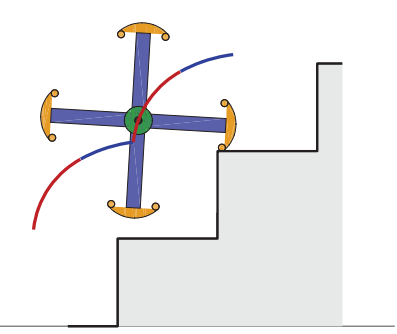

(b) Path of Umbrella Wheel on high stairs
Fig. 14 Path modeling of different stair rise/step height

Fig. 14 shows that the Umbrella Wheel can adapt easily to handle any staircase with dimensions within the proper range. 
The higher rise naturally requires larger extension of the arms, which can also be seen in Fig. 10.

\section{IMPLEMENTATION CONSIDERATIONS}

For the Umbrella Wheel to work properly, it is important that the vehicle that uses this concept is large enough so that it has a body that extends back from the wheel at least as far as the maximum extension of the arms (plus the radius of the wheel). Without this constraint there is a risk of the body spinning freely instead of turning the wheel. For smooth motion it is further more required that the center of gravity for the robot is also outside the reach of the wheel, otherwise the wheel might rotate with the body due to gravity. Additionally, the Umbrella Wheel requires knowledge about its own position as well as the position and dimensions of obstacles to be able to handle them optimally, so sensors are needed for a successful implementation of a vehicle using our concept.

\section{A. Size Limitations}

To ensure that the stair-climbing works, it is recommended that the radius of the wheel should be sufficiently small so that the wheel pieces fit on as many stairs as possible. The ground of a stair in publicly accessible areas has to, by the danish building regulations [9], be a minimum of $250 \mathrm{~mm}$, the American International Residential Code [10] state a minimum ground of $10 \mathrm{in}=25.6 \mathrm{~mm}$. The English Building Regulations [11] have a minimum of $220 \mathrm{~mm}$ in private areas and minimum of $250 \mathrm{~mm}$ for all public access stairs. Hence the following inequality should be fulfilled:

$$
r \cdot \frac{2 \pi}{n}+2 r_{o}+\pi r_{o}<G_{\text {min }}=250 \mathrm{~mm},
$$

although it can be recommended to have an extra margin for safety. Note that while there is an upper limit for step height, there is none for the ground and as such, any finite range of required extension cannot be given, though through modeling it can be tested beforehand wether a specific staircase can be climbed satisfactorily.

For a ladder-climbing scenario, it is important that the ladder does not prevent the wheel from turning. As such, the wheel must be small enough so that the widest part of the wheel segment is smaller than the gap between the rungs of the ladder. The following should be true if ladder-climbing is intended:

$$
\text { gap }>2 r_{o}+2\left(r-r_{o}\right) \cdot \sin \frac{\pi}{n}
$$

where an extra margin is also recommended for safety.

\section{B. Number of Segments}

The number of segments the Umbrella Wheel divides into affects how the wheel behaves on stairs and how many parts are required to construct it. Section IV has shown examples of stair-climbing behavior for different quantities of wheel segments. For smoother travel on stairs, more wheel segments are preferred, though for simpler construction considerations, fewer wheel segments are suitable.
Expression (14) shows that a larger number of wheel segments allows for a larger radius for the wheel in its closed configuration. The minimum tread size of the range of stairs targetted will condition both the closed radius of the wheel and the number of wheel segments.

For ladder-climbing, at least three wheel segments is recommended, otherwise the arms of the wheel could come into contact with the rungs of the ladder, though a design with two wheel segments could be designed to climb ladders as well.

The number of segments also affects the required extension for climbing stairs: if there are constraints on the wheel size, both with respect to radius/size in the open position and three-dimensional dimensions when closed, then a lower number of segments would be suitable.

\section{CONCLUDING REMARKS}

A new design for a stair-climbing wheel, referred to as Umbrella Wheel, has been proposed and discussed. The advantage of the concept lies in its ability to climb up or descend stairs, as well as go through rough terrain while being capable of regaining smooth displacement conditions on more level terrain. Simple considerations on geometry lead to modeling of the path followed by a single wheel. Different parameters of the wheel have been explored with regards to smoothness and versatility.

Current research includes the construction of an actuating mechanism in order to move the part responsible for the extension of each leg segment (part 2), as well as the design and development of a suitable vehicle to test the performance of the design with respect to stair-climbing as well as handling other obstacles and driving on smooth terrain.

\section{ACKNOWLEDGMENT}

This research was funded with support from the European Union Social Fund and the European Regional Development Fund.

\section{REFERENCES}

[1] US Patent Nr.: US255693 by J. G. TAUBER.

[2] R.C. Luo, M. Hsiao and T. Lin, "Errect Wheel-Legged Stair Climbing Robot for Indoor Service Applications", 2013 IEEE/RSJ International Conferrence on Intelligent Robots and Systems (IROS).

[3] P. Ben-Tzvi, S. Ito and A. Goldenberg, "Autonomous Stair Climbing with Reconfigurable Tracked Mobile Robot", ROSE 2007 - IEEE International Workshop on Robotic and Sensors Environments.

[4] Y. Sugahara, N. Yonezawa and K. Kosuge, "A Novel Stair-Climbing Wheelchair with Transformable Wheeled Four-Bar Linkages", The 2010 IEEE/RSJ International Conference on Intelligent Robots and Systems.

[5] D. Lu, E. Dong, C. Liu, M. Xu and J. Yang, "Design and Development of a Leg-Wheel Hybrid Robot "HyTRo-I"”, IROS 2013.

[6] C. Theeravithayangkura, T. Takubo, Y. Mae and T. Arai, "Stair Recognition with Laser Range Scanning by Limb Mechanism Robot "ASTERISK"", International Conference on Robotics and Biomimetics 2009.

[7] European Patent number EP2003039B1, "Trolley for ascending/descending stairs" by Alan Liva.

[8] A. Imadu, R. Tanaka, T. Kawai and M. Shibata, "Study on Design Methodology of Power-Assisted Luggage Cart with Rotational Arms for Stair Climbing", SICE Annual Conference 2011.

[9] Danish building regulations (Bygningsreglementet) 01.01.2016 30.06.2016 paragraph 3.2 .2

[10] International Residential Code, 2006 Stair Building Code.

[11] The Building Regulations Document K, 2013. 